\title{
Leptospira interrogans activation of peripheral blood monocyte glycolipoprotein demonstrated in whole blood by the release of IL-6
}

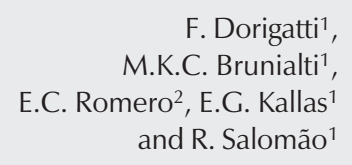

F. Dorigatti ${ }^{1}$,

M.K.C. Brunialti ${ }^{1}$,

E.C. Romero ${ }^{2}$, E.G. Kallas ${ }^{1}$

and R. Salomão ${ }^{1}$

\author{
1'Disciplina de Doenças Infecciosas e Parasitárias, Escola Paulista de Medicina, \\ Universidade Federal de São Paulo, São Paulo, SP, Brasil \\ ${ }^{2}$ Instituto Adolpho Lutz, São Paulo, SP, Brasil
}

\author{
Correspondence \\ R. Salomão \\ R. Pedro de Toledo, 781, 15ㅇandar \\ 04039-032 São Paulo, SP \\ Brasil \\ Fax: +55-11-5081-5394 \\ E-mail: \\ rsalomao-dipa@pesquisa.epm.br
}

Publication supported by FAPESP.

Received July 30, 2004 Accepted March 2, 2005

\begin{abstract}
Glycolipoprotein (GLP) from pathogenic serovars of Leptospira has been implicated in the pathogenesis of leptospirosis by its presence in tissues of experimental animals with leptospirosis, the inhibition of the Na,K-ATPase pump activity, and induced production of cytokines. The aims of the present study were to investigate the induction of IL-6 by GLP in peripheral blood mononuclear cells (PBMC) and to demonstrate monocyte stimulation at the cellular level in whole blood from healthy volunteers. PBMC were stimulated with increasing concentrations ( 5 to $2500 \mathrm{ng} / \mathrm{ml}$ ) of GLP extracted from the pathogenic $L$. interrogans serovar Copenhageni, lipopolysaccharide (positive control) or medium (negative control), and supernatants were collected after $6,20 / 24$, and $48 \mathrm{~h}$, and kept at $-80^{\circ} \mathrm{C}$ until use. Whole blood was diluted 1:1 in RPMI medium and cultivated for $6 \mathrm{~h}$, with medium, GLP and lipopolysaccharide as described above. Monensin was added after the first hour of culture. Supernatant cytokine levels from PBMC were measured by ELISA and intracellular IL-6 was detected in monocytes in whole blood cultures by flow-cytometry. Monocytes were identified in whole blood on the basis of forward versus side scatter parameters and positive reactions with CD45 and CD14 antibodies. GLP ( $\geq 50 \mathrm{ng} / \mathrm{ml}$ )-induced IL-6 levels in supernatants were detected after 6-h incubation, reaching a peak after 20/24 h. The percentage of monocytes staining for IL-6 increased with increasing GLP concentration. Thus, our findings show a GLPinduced cellular activation by demonstrating the ability of GLP to induce IL-6 and the occurrence of monocyte activation in whole blood at the cellular level.
\end{abstract}

Leptospirosis is a zooanthroponosis caused by pathogenic spirochetes of the genus Leptospira. Infection with these microorganisms may be nonsymptomatic or result in different
Key words

- Glycolipoprotein

- Leptospira interrogans

- Interleukin-6

- Monocytes

- CD45

- CD14 clinical conditions ranging from a "flu-like" disease with low morbidity to the severe or icteric form, also known as Weil's syndrome, which clinically resembles sepsis (1-3). 
Circulating levels of tumor necrosis factor alpha (TNF- $\alpha$ ) have been detected in patients with leptospirosis and are associated with the severity of the disease (4). Interleukin-10 (IL-10) levels were also elevated but were not associated with the severity of the disease in our previous work and a high IL$10 /$ TNF- $\alpha$ ratio was related to a better prognosis, suggesting that the anti-inflammatory response may be protective (5).

Several cellular components of $L$. interrogans, such as lipoproteins, lipopolysaccharide (LPS) and glycolipoprotein (GLP), are toxic and may participate in the pathogenesis of the disease (6). In experimental models with guinea pigs injected with pathogenic L. interrogans, GLP can be detected in the damaged tissues adhering to endothelial cells and to epithelial membranes, accompanying other antigen deposits resulting from bacterial destruction by the immune system (7-9). Therefore, the GLP complex may contain toxins involved in the pathogenesis of leptospirosis. In rabbit renal tubule epithelial cells, GLP inhibits sodium-potassium ATPase (Na,K-ATPase) pump activity in a dosedependent manner, a finding that may explain some electrolytic alterations observed in leptospirosis patients with acute renal failure (10). In a previous study we demonstrated that GLP could induce the production of TNF- $\alpha$ and IL-10 in peripheral blood mononuclear cells (PBMC) from healthy volunteers (11), although the cellular source of cytokines was not demonstrated.

The aims of the present study were to investigate the induction of IL- 6 by GLP in PBMC and to demonstrate monocyte stimulation at the cellular level in whole blood from healthy volunteers.

GLP was extracted from the pathogenic L. interrogans serovar Copenhageni obtained from a patient with Weil's syndrome by the method described by Vinh et al. (6). LPS from Salmonella abortus equi (kindly supplied by Dr. C. Galanos, Max Planck Institute of Immunobiology, Freiburg, Germany) was separated by the phenol-water method and purified by the phenol-chloroform-petroleum ether method (12). The study was approved by the University Ethics Committee and 18 healthy volunteers were included in the study after giving written informed consent to participate.

For the ELISA experiments, $10 \mathrm{ml}$ of peripheral venous blood was collected into heparin treated-tubes (Becton-Dickinson, Franklin Lakes, NJ, USA) and PBMC were obtained by the Ficoll gradient method (FicollPaque, Amersham Pharmacia Biotech, Uppsala, Sweden) and adjusted to $2 \times 10^{6}$ cells/ ml. PBMC were cultivated in RPMI 1640 medium (Sigma, St. Louis, MO, USA) supplemented with $10 \%$ fetal calf serum (HyClone, Logan, UT, USA) on 24-well plates (Nunclon; Nalge Nunc Int., Roskilde, Denmark). Cells were stimulated with $10 \mathrm{ng} / \mathrm{ml}$ LPS and 5 , 50, 500, and $2500 \mathrm{ng} / \mathrm{ml} \mathrm{GLP}$. Supernatants were collected at $6,20 / 24$, and $48 \mathrm{~h}$ of incubation and stored at $-80^{\circ} \mathrm{C}$ until use.

IL-6 was measured by capture ELISA. Antibody pairs and reagents were obtained from PharMingen (San Diego, CA, USA). Samples were tested in duplicate and a standard curve with human recombinant cytokine was constructed for each plate. Tests were performed according to manufacturer instructions.

For the detection of intracellular IL-6 in whole blood, $1 \mathrm{ml}$ of blood was diluted 1:1 in RPMI and incubated with $100 \mathrm{ng} / \mathrm{ml}$ LPS (positive control) and 5, 50, 250, and 500 ng/ $\mathrm{ml}$ GLP, or without a stimulus (negative control) for $1 \mathrm{~h}$ at $37^{\circ} \mathrm{C}$ in $5 \% \mathrm{CO}_{2}$. Monensin (Sigma) was added in a concentration of 2 $\mu \mathrm{M}$ and the samples were further incubated for $5 \mathrm{~h}$. Monoclonal antibodies were obtained from Becton Dickinson Immunocytometry Systems (BDIS, San Jose, CA, USA). The clones used, all obtained from BDIS, were: TNF- $\alpha$-fluorescein isothiocyanate (FITC), clone 6401.1111; CD14-perinidin chlorophyll protein (PerCP), cloneM $\phi \mathrm{P} 9$; IL-6phycoerythrin (PE), clone AS12; CD45-allo- 
phycocyanin O (APC), clone HI30. After incubation, CD14-PerCP and CD45-APC were added for $15 \mathrm{~min}$ at room temperature in the dark. Red blood cells were lysed, samples were washed with $2 \mathrm{ml}$ PBS, resuspended in $1 \mathrm{ml}$ fixation buffer (PBS and 4\% paraformaldehyde (Polyscience, Warrington, PA, USA), $\mathrm{pH}$ 7.4-7.6, and left to stand for $20 \mathrm{~min}$ at $4^{\circ} \mathrm{C}$ in the dark. The tubes were centrifuged, the supernatants discarded, and the cells resuspended in $0.3 \mathrm{ml}$ staining buffer (PBS with 1\% FCS (Gibco, Gaithersburg, MD, USA), $0.1 \%$ sodium azide (Sigma), $\mathrm{pH}$ 7.4-7.6, and stored at $4^{\circ} \mathrm{C}$ in the dark overnight. Cells were centrifuged and the supernatant was discarded. Samples were stained with $20 \mu \mathrm{l}$ monoclonal anti-IL-6-PE and $5 \mu 1$ monoclonal TNF- $\alpha$-FITC for 30 $\min$ at $4^{\circ} \mathrm{C}$ in the dark, washed in $2 \mathrm{ml}$ permeabilization buffer, resuspended in 0.3 $\mathrm{ml}$ staining buffer, and analyzed with a FACSCalibur flow-cytometer (BDIS).

Data acquisition and analyses were performed using CellQuest software (BDIS). Peripheral blood cells were acquired and monocytes were gated on the basis of CD14 and CD45 positivity and forward versus side scatter parameters. For each condition, 10,000 events were acquired. TNF- $\alpha-$ and IL-6-positive cells were reported as the percentage of monocytes (Figure 1).

The kinetics and dose-response of GLPinduced IL- 6 in PBMC were determined. Induction of IL- 6 was obtained with GLP concentrations of $50 \mathrm{ng} / \mathrm{ml}$ or higher $(\mathrm{P} \geq$ 0.05 GLP versus control). GLP-induced IL6 secretion was detected after $6 \mathrm{~h}$ of incubation, reaching a peak between 6 and $24 \mathrm{~h}$ of incubation and remaining stable after $48 \mathrm{~h}$ (Figure 2A).

Intracellular detection of IL-6 in monocytes was demonstrated after stimulating whole blood with GLP and LPS. The percentage of IL-6-positive monocytes increased with increasing GLP doses (Figure 2B).

In a previous study, we demonstrated that GLP extracted from the pathogenic $L$. interrogans serovar Copenhageni could induce PBMC activation. This was demonstrated by the secretion of TNF- $\alpha$ and IL-10 and the increase in the expression of CD69 and HLA-DR, markers of cellular activation. In the present study, we confirm and expand these results by demonstrating the secretion of IL-6 and monocyte activation at the cellular level in whole blood, through the intracellular detection of this cytokine.

LPS, peptidoglycan, and phospholipase are among the toxic components of $L$. interrogans that may play a role in the pathogenesis of leptospirosis (6,13-15). Lipoproteins are also potential toxic factors in leptospirosis as well as in other spirochetal diseases. Lipoproteins are recognized to be the most abundant proteins in spirochetes and are involved in disease pathogenesis through their ability to trigger the host inflammatory response (16). Expression of leptospiral lipoproteins Lip L32 and Lip L41 was demonstrated by immunohistochemistry of kidney tissue following infection with virulent $L$. kirschneri. Treponemal and Borrelial lipoproteins are capable of activating monocytes in vitro and cellular activation has been shown to involve CD14 and Toll-like receptor 2 molecules (16-18).

GLP is likely to be involved in the pathogenesis of the disease as suggested by the presence of GLP in damaged tissues of patients and experimental animals (19). Moreover, it was demonstrated that this component might block the tubular epithelial cell sodium pump in the kidneys (10). This may be one of the mechanisms of renal dysfunction that is characteristic of the disease. Other toxic effects exerted by GLP may be observed in cultures of Vero and L929 cells, leading to cell membrane perforations followed by cell death (6).

The toxic effects of bacterial compounds, such as the Gram-negative outer membrane LPS, are mediated by the interaction with host cells, with a central role for macrophages (12). Macrophage-derived cytokines, such 
Figure 1. Detection of intracellular cytokines in monocytes following whole blood stimulation with GLP. Whole blood was stimulated for $6 \mathrm{~h}$ with LPS, GLP or not, the last $5 \mathrm{~h}$ in the presence of monensin. Monocytes were gated combining the forward scatter versus side scatter parameters, with a gate over the cells with low to intermediate size and granularity (R1), and CD14- and CD45stained cells (R2) (A), and the TNF- $\alpha$ - and IL-6-stained cells are reported as the percentage of monocytes (B). Quadrant statistics were established using cells with isotype monoclonal antibodies. GLP = glycolipoprotein; LPS = lipopolysaccharide.
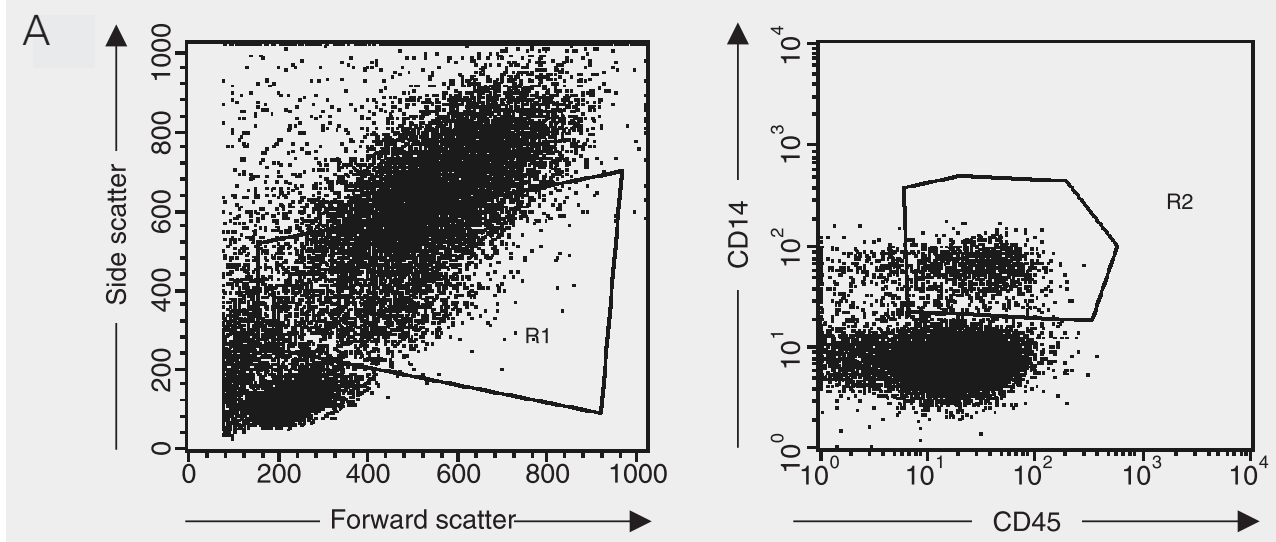

B
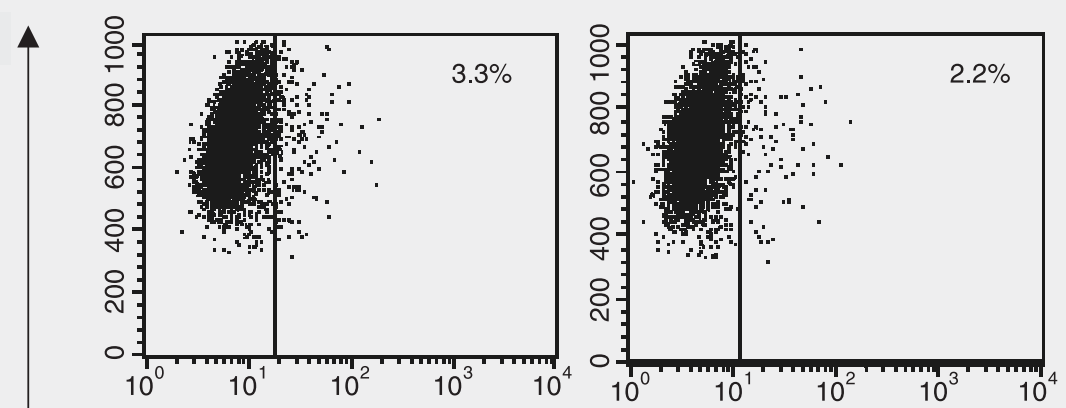

Control
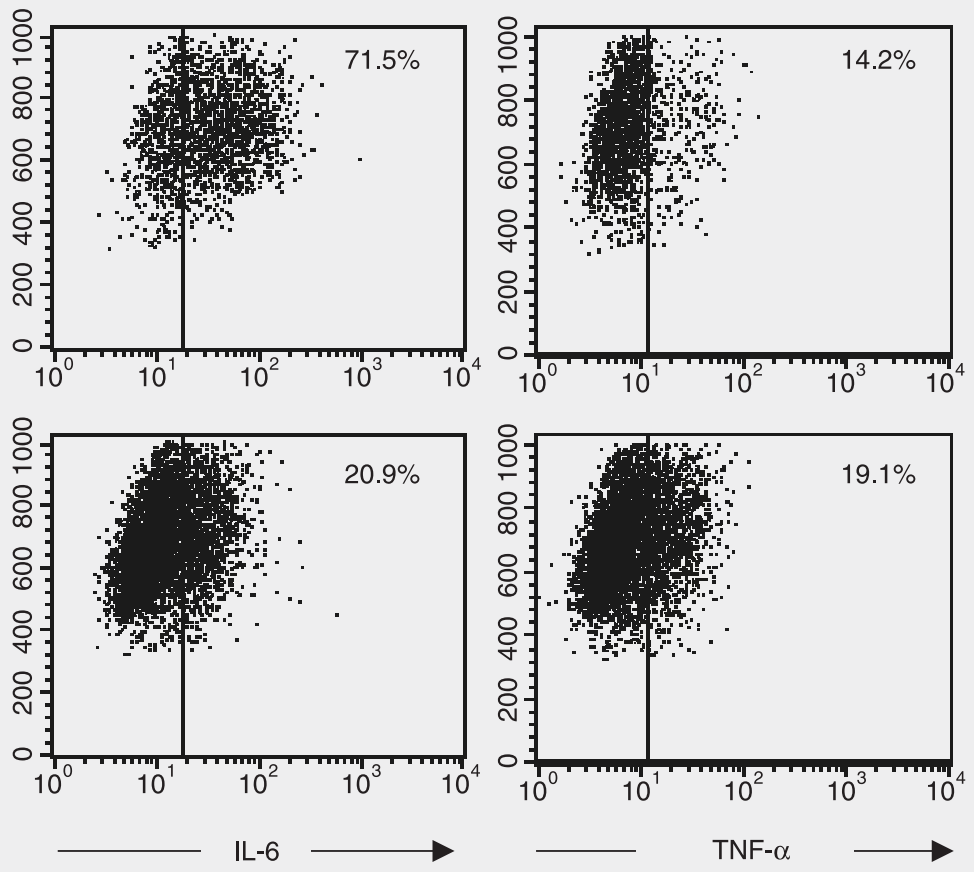

LPS

GLP 


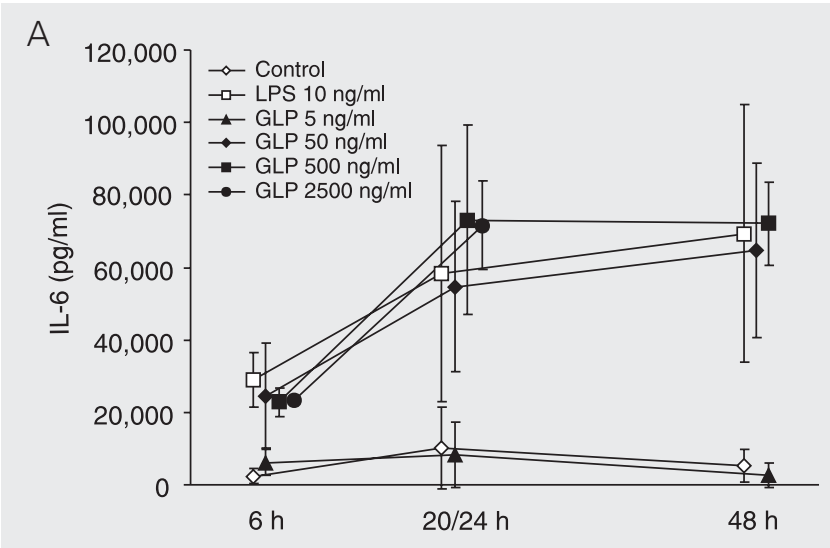

B

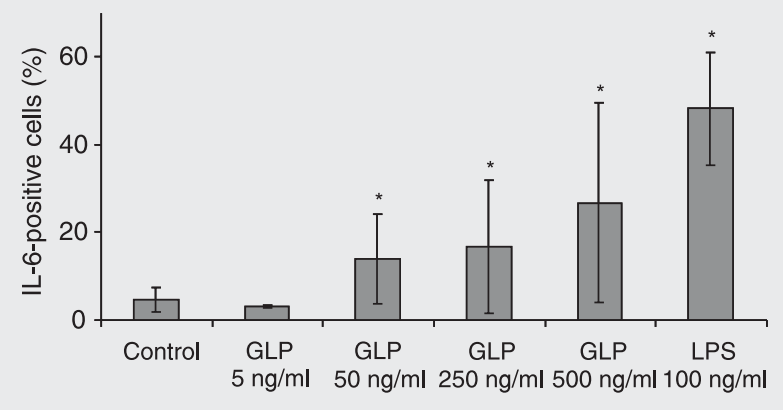

Figure 2. Kinetics of IL-6 release (A) and detection of IL-6 in monocytes in whole blood after stimulation with GLP (B). A, LPS was used as positive control. Peripheral blood mononuclear cells were stimulated with either Salmonella abortus equi LPS or Leptospira interrogans serovar Copenhageni GLP at the doses indicated and incubated for $48 \mathrm{~h}$. Supernatants were collected after 6, 20/24, and $48 \mathrm{~h}$ and IL-6 was measured by ELISA. Results are reported as the mean \pm SD for three to five experiments. GLP $=$ glycolipoprotein; LPS $=$ lipopolysaccharide. GLP $(\geq 50 \mathrm{ng} /$ $\mathrm{ml}$ ) versus control: $\mathrm{P}<0.05$ for 6 and $20 / 24 \mathrm{~h}$ and $\mathrm{P}=0.08$ for $48 \mathrm{~h}$ (Mann-Whitney test). $B$, The cytokine-producing cells are reported as the percentage of monocytes. Data are reported as means \pm SD for six experiments. ${ }^{*} \mathrm{P}<0.05$ versus control (Mann-Whitney test).

as TNF- $\alpha$ and IL-6, are considered to be mediators of LPS toxicity, yet fundamental for the host defense against microorganisms (12). Circulating levels of TNF- $\alpha$ have been demonstrated in patients with leptospirosis and their presence is associated with poor outcomes (4). The presence of IL-6 in PBMC supernatants following GLP stimulation ex- pands the array of cytokines induced by this toxin. The detection of intracellular IL-6 in monocytes following whole blood stimulation with GLP is a direct demonstration of monocyte activation and provides evidence for the participation of GLP in the pathogenesis of leptospirosis.

\section{References}

1. Doenças Infecciosas e Parasitárias. Aspectos Clínicos, Vigilância Epidemiológica e Medidas de Controle (2000). FUNASA, 208 (Guidelines).

2. Faine S (1994). Leptospira and Leptospirosis. CRC Press, Boca Raton, FL, USA (Guidelines).

3. Lomar AV, Veronesi R, Brito T \& Diament D (1997). Leptospiroses. In: Veronesi R \& Focaccia R (Editors), Tratado de Infectologia. Atheneu, São Paulo, SP, Brazil.

4. Tajiki MH \& Salomão R (1996). Association of plasma levels of tumor necrosis factor $\alpha$ with severity of disease and mortality among patients with leptospirosis. Clinical Infectious Diseases, 23: 1177-1178.

5. Tajiki MH, Nakama AS \& Salomão R (1997). The ratio of plasma levels of IL-10/TNF- $\alpha$ and its relationship to disease severity and survival in patients with leptospirosis. Brazilian Journal of Infectious Diseases, 1: 138-141.

6. Vinh T, Adler B \& Faine S (1996). Glycolipoprotein cytotoxin from Leptospira interrogans serovar copenhageni. Journal of General Microbiology, 132: 111-123.

7. Alves VAF, Gayotto LC, Yasuda PH, Wakamatsu A, Kanamura CT
\& De Brito T (1991). Leptospiral antigens (L. interrogans serogroup icterohaemorrhagiae) in the kidney of experimentally infected guinea pigs and their relation to the pathogenesis of the renal injury. Experimental Pathology, 42: 81-93.

8. Alves VA, Gayotto LC, De Brito T, Santos RT, Wakamatsu A, Vianna MR \& Sakata EE (1992). Leptospiral antigens in the liver of experimentally infected guinea pig and their relation to the morphogenesis of liver damage. Experimental and Toxicologic Pathology, 44: 425-434.

9. Pereira MM, Andrade J, Lacerda MD, Batoreu MM, Marchesvky RS \& Ribeiro dos Santos R (1997). Demonstration of leptospiral antigens on tissues using monoclonal antibodies and avidin-biotin peroxidase staining. Experimental and Toxicologic Pathology, 49: 505-511.

10. Younes-Ibrahim M, Burth P \& Faria MVC (1995). Inhibition of Na,KATPase by endotoxin extracted from Leptospira interrogans: a possible mechanism for the physiopathology of leptospirosis. Comptes Rendus de L'Académie des Sciences, 318: 619-625.

11. Diament D, Brunialti MKC, Romero EC, Kallas EG \& Salomao R (2002). Peripheral blood mononuclear cell activation induced by Leptospira interrogans glycoprotein. Infection and Immunity, 70: 
1677-1683.

12. Galanos C, Frendenberg MA, Katschinski R, Salomão R, Mossmann H \& Kumazawa $Y$ (1992). Tumor necrosis factor and host response to endotoxin. In: Ryan JL \& Morrison DC (Editors), Bacterial Endotoxin Lipopolysaccharides. Immunopharmacology and Pathophysiology. CRC Press, Boca Raton, FL, USA.

13. Cinco M, Vecile E \& Murgia R (1996). Leptospira interrogans and Leptospira peptidoglycans induce the release of tumor necrosis factor- $\alpha$ from human monocytes. FEMS Microbiology Letters, 138: 211-214.

14. Isogai E, Isogai H, Kurebayashi $Y$ \& Ito $N$ (1986). Biological activities of Leptospiral lipopolysaccharide. Zentralblatt für Bakteriologie, Mikrobiologie und Hygiene, Series A, 261: 53-64.

15. Yanagihara $Y$, Taniyama $T$ \& Misaki $H$ (1984). Phospholipases of Leptospira. I. Presence of phospholipase A1 and Iysophospholipase in Leptospira biflexa. Microbiology and Immunology, 28: 747-756.
16. Haake DA, Chao G, Zuerner RL \& Barnett JK (2000). The leptospiral major membrane protein Lip L32 is a lipoprotein expressed during mammalian infection. Infection and Immunity, 68: 22762285.

17. Werts C, Tapping RI \& Mathison JC (2001). Leptospiral lipopolysaccharide activates cells through a TLR2-dependent mechanism. Nature Immunology, 4: 346-352.

18. Wooten RM, Morrison TB, Weis $\mathrm{JH}$, Wright SD, Thieringer R \& Weiss JJ (1998). The role of CD14 in signaling mediated by outer membrane lipoproteins of Borrelia burgdorferi. Journal of Immunology, 160: 5485-5492.

19. Macedo-Santos RT, Sakata EE \& Yasuda PH (1989). Glicoproteína de Leptospira interrogans sorogrupo icterohaemorrhagiae: distribuição em fígado e rim de cobaias experimentalmente infectadas. Revista do Instituto de Medicina Tropical, 31: 235241. 